\title{
Comparative Analysis of Performance of PREVI Fluo TB Method by Using LED-Microscopy
}

Semaria Solomon ${ }^{1 *}$, Solomon Gebreselassie ${ }^{2}$, Berhanu yitayew ${ }^{3}$ and Abebaw Kebede ${ }^{4}$

${ }^{1}$ St. Paul's Hospital Millennium Medical College, Addis Ababa, Ethiopia

${ }^{2}$ Department of Microbiology, Immunology, and Parasitology, Addis Ababa University, Ethiopia

${ }^{3}$ Institute of medicine and health sciences, college of medicine, Debre Brehan University, Ethiopia

${ }^{4}$ Ethiopian Public Health Institute, Addis Ababa, Ethiopia

\begin{abstract}
There is a need for rapid, practical and accurate tuberculosis diagnostic tools that are adapted to resource-poor settings in order to ensure that those affected receive proper and timely treatment. Light-Emitting-Diode microscopy (LED) has recently been endorsed by the WHO for diagnosis of TB in these countries. A much recent smear diagnosis method, PREVI Fluo TB by using LED microscopy, has been made available. However, due to the novelty of the method, there is no information available on the specificity and sensitivity when compared to established methods such as the ordinary LED-Auramine $\mathrm{O}$ or the classical widely used ZiehlNeelsen (ZN) in TB positive persons. This study was undertaken to compare the sensitivity, specificity, positive predictive value and negative predictive value of PREVI Fluo TB stain with ZN method and Auramine O-LED microscopy taking culture as reference. A prospective cross sectional study was conducted in St. Paul's Hospital Millennium Medical College, Addis Ababa, Ethiopia. SpotMorning-Spot sputum samples from 248 TB suspected study participants were collected. The smear detection rate of PREVI Fluo TB, ZN, Auramine O and culture were found to be $35(14.1 \%), 24(9.7 \%), 44(17.7 \%)$ and $30(12.1 \%)$ respectively. The sensitivity of the PREVI Fluo TB method was $76.67 \%$, better than ZN and slightly less than Auramine O which were $59.07 \%$ and $78.13 \%$ respectively. Nevertheless, the specificity (94.5\%) was lower than ZN (96.79\%) and higher than Auramine O (91.2\%). The negative likelihood ratio of PREVI Fluo TB, ZN, and Auramine O methods were $0.25,0.45$, and 0.24 respectively. The PREVI Fluo TB method had a PPV of $65.71 \%$ which is higher than the Auramine O (56.82\%) but with a comparable NPV $(96.71 \%$ and $96.57 \%$ respectively). The respective agreements of the $\mathrm{ZN}$, Auramine $\mathrm{O}$ and PREVI Fluo TB methods with the gold standard were $\mathrm{K}=0.585, \mathrm{~K}=0.621$ and $\mathrm{K}=0.664$. There was a substantial agreement of PREVI Fluo TB result with $Z N(k=0.636)$ and Auramine $O$ methods $(K=0.745)$. Given the practical benefits of PREVI Fluo TB for TB diagnosis, and comparable accuracy to the current standard of Auramine O fluorescence method and the gold standard culture, PREVI Fluo TB should be considered by TB diagnostic laboratories, as an alternative diagnostic tool for conventional Auramine $O$ fluorescent stain.
\end{abstract}

\section{Keywords: Tuberculosis; ZN; Auramine O; PREVI Fluo TB}

\section{Introduction}

Tuberculosis (TB) is one of the oldest known human diseases and it is still one of the major causes of mortality and a major public health problem particularly in low-income countries. It is estimated that nearly one billion people will be infected with TB, 200 million develop the disease, and 35 million will die from TB during 20002020 [1]. Different diagnostic methods are available for the diagnosis of pulmonary tuberculosis. The "gold standard" of TB diagnosis is identification of the bacilli after culturing it and growing sufficient quantities for analysis. However, culturing the TB bacteria is expensive and slow (taking weeks), and requires technical facilities that are often unavailable in developing countries. Thus, diagnosis is often based on a test for "acid-fast" bacteria in a sputum smear (made by smearing sputum on a microscope slide). In patients with active pulmonary TB, only an estimated $45 \%$ of infections are detected by sputum microscopy [2]. This test (Ziehl-Neelsen) has the advantage of being simple but is hampered by very low sensitivity; it may only detect half of all cases with active infection. It is also very dependent on the skill of the technician [3]. Specificity of ZN microscopy is high but sensitivity is variable $(20-80 \%)$ and significantly reduced in extra-pulmonary TB and in HIV-infected TB patients [4]. Besides being labour-intensive, direct sputum smear microscopy may have considerable patient costs and inconvenience associated with the need to submit multiple sputum specimens over a period of up to three days [5].

A recently developed conventional fluorescent microscopy has documented higher sensitivity than $\mathrm{ZN}$ and takes less time, but uptake has been hampered by high cost due to expensive mercury vapour light sources, the need for regular microscopy maintenance, and the requirement for a dark room. Light emitting diode (LED) technology has been developed over recent years to allow the benefits of fluorescent microscopy without the associated costs [6]. Light emitting diode (LED) microscopy is a novel diagnostic tool developed primarily to allow resource-poor parts of the world access to the benefits of FM [7]. Compared to conventional mercury vapour fluorescence microscopes, LED microscopes are less expensive and have lower maintenance requirements. The diodes are very durable, do not require warm-up time, and do not contain toxic products [8]. Importantly, they are reported to perform equally well without a darkroom. These qualities make them attractive for use in low- and middle-income countries, and they have performed well in evaluations in these settings [9].

WHO recommend that conventional fluorescent microscopy must be replaced by LED microscopy and that LED microscopy be phased in as an alternative for conventional $\mathrm{ZN}$ light microscopy. Countries implementing LED microscopy should address laboratory staff training, country validation, introduction of appropriate quality assurance, and monitoring of impact on TB case detection and treatment outcome [6]. PREVI Fluo TB is the new and promising method for the diagnosis of PTB which is developed by Biomerieux SA. The method has similar principle with the conventional LED fluorescent microscope except that it has a fixative, decolorizer ethanol than the more toxic methanol

*Corresponding author: Semaria Solomon,, St. Paul's Hospital Millennium Medical College, Addis Ababa, Ethiopia P. O. Box 25181; Tel: +251 911217830; E-mail: semariasol@gmail.com

Received February 13, 2015; Accepted April 21, 2015; Published April 23, 2015

Citation: Solomon S, Gebreselassie S, Yitayew B, Kebede A (2015) Comparative Analysis of Performance of PREVI Fluo TB Method by Using LED-Microscopy. J Med Microb Diagn 4: 178. doi:10.4172/21610703.1000184

Copyright: (c) 2015 Solomon S, et al. This is an open-access article distributed under the terms of the Creative Commons Attribution License, which permits unrestricted use, distribution, and reproduction in any medium, provided the original author and source are credited. 
and a red Thiazine Red counter staining dye. The fixative helps to fix the smear to the slide so that there would not be cross contamination and environmental contaminant. However, it needs to see its diagnostic efficacy with other commonly used diagnostic methods. Therefore, this study was designed to compare three methods of microscopy stains; the Ziehl-Neelsen, Auramine O and PREVI fluo TB taking culturing as a gold standard by using well characterized clinical specimens from patients who were assessed according to standard parameters for $M$. tuberculosis.

\section{Methods}

A hospital based comparative cross sectional study was conducted at St. Paul's hospital Millennium Medical College, Addis Ababa, Ethiopia. All patients who were suspected for pulmonary tuberculosis during the data collection period were included. In this study 248 study participants were included. Three sputum samples were collected from each participants (spot-morning-spot). For every sample taken 3 smears were made for $\mathrm{ZN}$, Auramine-O and PREVI Fluo TB stains. Hence, for every study participants a total of 9 slides were made, 3 for every sample. ZN smears were examined at 1,000× magnification, and fluorescent smears (in accordance with the International Union Against Tuberculosis and Lung Disease Working Group on Smear Microscopy) at $200 \times$, with confirmation of positive smears at $400 \times$ magnification. Each of the microscopy readings were made without knowledge of the results of each slide and all microscopic readings were made without knowledge of the culture result. Smears were classified as positive when $\geq 1$ AFB was detected per 100 fields, and patients were considered smear-positive if they had $\geq 1$ positive smear [10]. The main outcomes for comparison were the sensitivity and specificity of PREVI Fluo compared with the sensitivity and specificity of $\mathrm{ZN}$ and Auramine $\mathrm{O}$ stains using LED microscope for the fluorescent stains and light microscopy for $\mathrm{ZN}$. Culture was used as the reference standard. Sensitivity, specificity, positive and negative predictive values and likelihood ratios were calculated by using OpenEpi Version 2.3 for the PREVI Fluo LED, Auramine O LED and conventional ZN microscopy using mycobacterial culture as the reference standard. The agreement of PREVI Fluo TB method with the reference culture and its agreement with the ZN and Auramin O was calculated using Kappa statistics.

\section{Results}

A total of 248 pulmonary tuberculosis suspected patients were included and from which $25.4 \%$ (63/248) had previous pulmonary tuberculosis history contact, while the remaining $74.6 \%$ had no history of previous pulmonary tuberculosis contact.

Laboratory investigation of every collected sputum samples of the study participants were done by the three methods and confirmed with the forth gold standard method TB culture. A total of 2,232 slides were made for ZN, Auramine O and PREVI Fluo methods, 744 for each type of method and 248 slides for each Spot-Morning-spot sample. By ZN method out of the total study participants $24(9.7 \%)$ were positive. In Auramine O 44(17.7\%) were positive while in PREVI Fluo TB method, 35 (14.1\%) participants were positive. From the sputum of the participants cultured on LJ culture media, 30(12.1\%) of the participants became positive for TB bacilli whereas the remaining 213(85.9\%) were negative (Table 1).

Grading of bacilli (Scanty, $1+, 2+$ and $3+$ ) were also performed to see the differences among each method. For instance in the PREVI Fluo method, in 'slide a ( $1^{\text {st }}$ spot)' 3 of the smears $(1.2 \%)$ had a $1+$ result, 3 $(1.2 \%)$ had $2+, 10(4 \%)$ had a $3+$ and $10(4 \%)$ had a scanty result. In 'slide b (morning)' 4 of the smears (1.6\%) had a $1+$ result, $4(1.6 \%)$ had $2+, 10(4 \%)$ had a $3+$ and $10(4 \%)$ had a scanty result. And at last, in 'slide $\mathrm{c}$ (second spot) 6 of the smears $(2.4 \%)$ had a $1+$ result, $3(1.2 \%)$ had $2+, 8(3.2 \%)$ had a $3+$ and $14(5.6 \%)$ had a scanty result (a spot, $\mathrm{b}$ morning and $\mathrm{c}$ spot). The bacilli detected in culture from positive finding were graded as $23.3 \%(7 / 30), 10 \%(3 / 30), 33.3 \%(10 / 30)$ and $33.3 \%(10 / 30)$ for $1+, 2+, 3+$ and scanty respectively (Table 2$)$.

In a cross tabulation made between PTB culture result and the different methods, of the total $24 \mathrm{Zn}$ positive slides $17(70.8 \%)$ were positive with LJ TB culture while 7(29.2\%) were negative with culture and $13(5.8 \%)$ of the total culture positives were negative with $\mathrm{ZN}$. Among the total 34 Auramine O positive slides, 25(56.8\%) were positive with culture, the rest 19(43.2\%) were negative with culture and 5(2.5\%) of the total culture positives gave negative results with Auramine O. Within the total 35 PREVI Fluo positive slides, 23(65.7\%) were also positive with culture, $12(34.3 \%)$ were negative and $7(3.3 \%)$ of the culture positives, gave negative result with PREVI fluo method. Concerning the agreement of the three methods with the gold standard (LJ culture), $\mathrm{ZN}$ has a kappa agreement result of $K=0.585$, for Auramine $O K=0.621$ and PREVI Fluo TB K=0.664 (Table 3).The PREVI Fluo TB method has an agreement with the kappa scale of 0.745 with the Auramine O method and $\mathrm{K}=0.636$ with regards to its agreement with the $\mathrm{ZN}$ method (Table 3). PTB positive results with the different stains related to HIV positive study participants is shown in the Table 4 . Four (9.3\%) of the total 43 HIV positives, were also positive for $\mathrm{ZN}$ smear, six (14\%) were positive with Auramine $\mathrm{O}$ and 5 (11.6\%) were positive with PREVI Fluo TB.

The sensitivity and Specificity of ZN, Auramine O and PREVI Fluo were $56.67 \%$ and $96.79 \%, 78.13$ and $91.2 \%$ and $76.67 \%$ and $94.5 \%$ respectively. The different tests gave a PPV and NPV results of $70.83 \%$ and $94.2 \%(\mathrm{ZN}), 56.82 \%$ and $96.57 \%$ (Auramine $\mathrm{O}$ ) and $65.71 \%$ and 96.71\% PREVI Fluo methods. In addition, the diagnostic accuracy for the different tests were $91.94,89.52$ and $92.34 \%$ respectively for $\mathrm{ZN}$, Auramine $\mathrm{O}$ and PREVI Fluo TB. As well the likely hood ratio for a negative test was calculated to be $0.44,0.23$ and 0.24 for these tests (Table 5).

\begin{tabular}{|c|c|c|}
\hline Methods & PTB Lab result Positive Negative No. (\%) No. (\%) & Total No. (\%) \\
\hline ZN & 24(9.7) 224(90.3) & $248(100)$ \\
\hline Auramine O & $44(17.7) 204(82.3)$ & $248(100)$ \\
\hline PREVI Fluo & $35(14.1) 213(85.9)$ & $248(100)$ \\
\hline Culture & $30(12.1) 218(87.9)$ & $248(100)$ \\
\hline
\end{tabular}

Table 1: Results of the $Z N$, Auramine $O$ and PREVI Fluo TB methods in a comparative study of PREVI Fluo TB method among TB suspected patients.

\begin{tabular}{|l|c|c|c|}
\hline WHO grading scale & ZN Freq (\%) & Auramine O Freq (\%) & PREVI Fluo Freq (\%) \\
\hline Slide A(1 ${ }^{\text {st }}$ spot) Scanty & $3(1.2)$ & $14(46.7)$ & $10(4)$ \\
\hline $1+$ & $8(3.2)$ & $4(13.3)$ & $3(1.2)$ \\
\hline $2+$ & $2(0.8)$ & $4(13.3)$ & $3(1.2)$ \\
\hline $3+$ & $6(2.4)$ & $8(26.7)$ & $10(4)$ \\
\hline Slide B(morning)Scanty & $3(1.2)$ & $12(4.8)$ & $10(4)$ \\
\hline $1+$ & $6(2.4)$ & $6(2.4)$ & $4(1.6)$ \\
\hline $2+$ & $4(1.6)$ & $4(1.6)$ & $4(1.6)$ \\
\hline $3+$ & $4(1.6)$ & $8(3.2)$ & $10(4)$ \\
\hline Slide C (2nd spot) Scanty & $14(5.6)$ & $12(4.8)$ & $14(5.6)$ \\
\hline $1+$ & $4(1.6)$ & $4(1.6)$ & $6(2.4)$ \\
\hline $2+$ & $4(1.6)$ & $6(2.4)$ & $3(1.2)$ \\
\hline $3+$ & $8(3.2)$ & $7(2.8)$ & $8(3.2)$ \\
\hline
\end{tabular}

Table 2: Grading results of PTB positive results in $\mathrm{ZN}$, Auramine $\mathrm{O}$ and PREV Fluo TB methods based on WHO grading system, 2012. 


\begin{tabular}{|c|c|c|c|}
\hline Variables & \multicolumn{2}{|c|}{$\begin{array}{l}\text { Culture confirmed TB } \\
\text { result Positive Negative } \\
\text { Yes (\%) No (\%) }\end{array}$} & $\begin{array}{l}\text { Agreementwith } \\
\text { Kappa }\end{array}$ \\
\hline \multicolumn{4}{|c|}{ ZN TB result } \\
\hline Positive & $17(70.8)$ & $7(29.2)$ & \multirow{2}{*}{0.585} \\
\hline Negative & $13(5.8)$ & 211 (94.2) & \\
\hline \multicolumn{4}{|c|}{ Auramine O TB result } \\
\hline Positive & $25(56.8)$ & $19(43.2)$ & \multirow{2}{*}{0.621} \\
\hline Negative & $5(2.5)$ & $199(97.5)$ & \\
\hline \multicolumn{4}{|c|}{ PREVI Fluo TB result } \\
\hline Positive & $23(65.7)$ & $12(34.3)$ & \multirow{2}{*}{0.664} \\
\hline Negative & $7(3.3)$ & 206 (96.7) & \\
\hline
\end{tabular}

Table 3: PTB culture result related to ZN, Auramine $O$ and PREVI Fluo for comparative analysis of PREVI Fluo among TB suspected patients.

\begin{tabular}{|c|c|c|c|}
\hline PREVI Fluo TB result & \multicolumn{2}{|c|}{$\begin{array}{l}\text { Auramine O PTB } \\
\text { resultPositive NegativeYes } \\
(\%) \text { No (\%) }\end{array}$} & $\begin{array}{l}\text { Agreement with } \\
\text { Kappa }\end{array}$ \\
\hline \multicolumn{4}{|c|}{ PREVI Fluo TB result } \\
\hline Positive Count & $31(88.6)$ & $4(11.4)$ & 0.745 \\
\hline Negative Count & $13(6.1)$ & $200(93.9)$ & \\
\hline \multicolumn{4}{|l|}{ ZN PTB result } \\
\hline \multicolumn{4}{|c|}{ PREVI Fluo TB result } \\
\hline Positive Count & $20(57.1)$ & $15(42.90$ & \multirow{2}{*}{0.636} \\
\hline Negative Count & $4(1.9)$ & $209(98.1)$ & \\
\hline
\end{tabular}

Table 4: Agreement of Kappa statistics of PREVI Fluo TB with Auramine O for comparative analysis of PREVI Fluo among TB suspected patients.

\begin{tabular}{|l|l|l|l|}
\hline & ZN \% (95\%CI) & $\begin{array}{l}\text { Auramine O\% } \\
\text { (95\%CI) }\end{array}$ & $\begin{array}{l}\text { PREVI Fluo\% } \\
\mathbf{( 9 5 \% C I ) ~}\end{array}$ \\
\hline Sensitivity & $56.67(39.2-72.62)$ & $78.13(61.24-88.98)$ & $76.67(59.07-88.21)$ \\
\hline Specificity & $96.79(93.52-98.44)$ & $91.28(6.67-94.3)$ & $94.5(90.63-96.82)$ \\
\hline $\begin{array}{l}\text { Positive Predictive } \\
\text { value }\end{array}$ & $70.83(50.83-85.09)$ & $56.82(42.22-70.32)$ & $65.71(49.15-79.17)$ \\
\hline $\begin{array}{l}\text { Negative predictive } \\
\text { value }\end{array}$ & $94.2(90.33-96.58)$ & $96.57(93.03-98.33)$ & $96.71(993.37-98.4)$ \\
\hline Diagnostic Accuracy & $91.94(87.87-94.72)$ & $89.52(85.08-0.92)$ & $92.34(88.34-95.04)$ \\
\hline $\begin{array}{l}\text { Likely hood ratio of } \\
\text { a Negative test }\end{array}$ & $0.44(0.38-0.52)$ & $0.23(0.18-0.31)$ & $0.24(0.19-0.33)$ \\
\hline
\end{tabular}

Table 5: The sensitivity, specificity, PPV, NPV, diagnostic accuracy and likely hood ratio, for the assessment for comparative analysis of PREVI Fluo.

\section{Discussion}

The smear positivity rate of PTB by ZN, Auramine O and PREVI Fluo TB methods were investigated. In $\mathrm{ZN}$ method, out of the total study participants, 9.7\%, in Auramine O $17.7 \%$ and in PREVI Fluo TB method $14.1 \%$ participants were positive for at least one slide. This has a similar finding from Vietnam where the prevalence of smearpositive TB by ZN method was 7\% [11]. The sensitivity and specificity of ZN, PREVI Fluo and Auoramine O were 56.67\% (39.2-72.62) and 96.79\% (93.52-98.44), 76.67\% (59.07-88.21) and 94.5\% (90.63-96.82) and $78.13 \%(61.24-88.98)$ and $91.2 \%(86.67-94.3)$ respectively. ZN method was the least sensitive with highest specificity compared to the two methods. This is in line with Studies using Auramine O for comparison with conventional microscopy where sensitivity of conventional microscopy ranged from 0.48 to 0.93 , and from 0.57 to 0.93 for fluorescence microscopy [12]. The decreased magnification used during LED microscopy, compared with light microscopy may also have contributed, particularly toward the sensitivity differences noted. This is similar to the study in Iran where the $\mathrm{ZN}$ sensitivity was found to be $51 \%$ lower than the sensitivity of Auramine O (57\%) [13]. Again the finding of the current study is in agreement with the study conducted in Ethiopia, Kenya and Tanzania which used three spotmorning-spot sputum sample for the comparison of LED-FM and $\mathrm{ZN}$ with findings of $77 \%$ and $70.5 \%$ of sensitivity and $88 \%$ and $96.5 \%$ specificity respectively [14]. It is also in agreement with the study done in Turkey where the ZN sensitivity was much lower than the FM. The ZN sensitivity was found to be $67.6 \%$ and the FM was $85.2 \%$ [15]. Still Another study done in Lagos, Nigeria on the sensitivity of direct smear microscopy gave a sensitivity of $53.8 \%$ by the $\mathrm{ZN}$ method and gave a much lower sensitivity of ZN for HIV positive individuals [16].

Moreover, this study is in agreement with the study by Torrea et al, (2008) who reported FM detected on average 18\% more positives than $\mathrm{ZN}$. In addition, there was a substantial agreement with ZN TB smear positivity result and PREVI Fluo TB methods $(\mathrm{K}=0.636)$. Out of the total 35 PREVI Fluo positive cases, $20(57.1 \%)$ had also a positive result with the Auramine O, while 14 had a discordant result. Out of $9.7 \%$ of the $\mathrm{ZN}$ total cases $1.9 \%$ were negative with PREVI Fluo.

The smear negativity rate of this study was seen in $\mathrm{ZN}$ stained slides from which $24(9.7 \%)$ were positive by $\mathrm{ZN}$ and $30(12.1 \%)$ were confirmed culture positive. Out of these 6 of the slides which were missed by $\mathrm{ZN}$ were positive by culture. Therefore the $\mathrm{ZN}$ miss diagnosed as a negative $2.4 \%$ of the confirmed positive cases. In a similar study in Vietnam the smear negativity was reported to be $2 \%$ [11]. For those patients with a high clinical suspicion, clinicians must face the dilemma of empirically treating or waiting for up to 8 weeks for the final culture results. These newer fluorescent diagnostic tests are most suitable in identifying these smear negative cases, as they did not miss any of these cases in this study.

The detection rate in $\mathrm{ZN}$ was also lower than the new PREVI Fluo methods with a smear detection rate of $9.7 \%$ and $14.1 \%$ respectively. The detection rate of bacilli by $\mathrm{ZN}$ method is lower in HIV positive individuals [17] similarly in the current study $\mathrm{ZN}$ is slightly lower than Auramine $\mathrm{O}$ and PREVI Fluo.

The sensitivity and specificity of $\mathrm{ZN}$ method were $56.7 \%$ and $96.8 \%$ respectively with culture taken as a gold standard and PPV, NPV and diagnostic accuracy were found to be $70.8 \%, 94.2 \%$ and $91.9 \%$ correspondingly. Sensitivity of ZN was lower than any other methods. This is in line with the report by Khatun et al., (2011) found that the sensitivity of and specificity of ZN were lower than LED microscope which were $56.06 \%$ and $97.61 \%$ respectively, also in a similar study in the Netherlands with regards to $\mathrm{ZN}, 61.1 \%$ and $98.9 \%$ sensitivity and specificity were found respectively [18]. The sensitivity of $\mathrm{ZN}$ in this study was lower than that found in Tanzania (61.8\%) [19]. Reasons for this could be that the concentrated smear method was used for the study in Tanzania and all study participants were HIV positive. However, the finding is in contrast with the report by Maryline et al. (2011) who found a sensitivity of $72 \%$, this might be due to whether the Ziehl Neelsen staining method is always done first, as might be required in a diagnostic setting. A factor that may contribute to variability in the findings between these studies is of study participant difference. Again in their study all the study participants were only HIV positives, but in the current study only 43 HIV positive individuals were included. Similar finding to this study was also reported from India which was $55.55 \%$ sensitivity of ZN and $71.85 \%$ sensitivity of Auramine O. Direct fluorescent microscopy detected $9.29 \%$ paucibacillary sputum samples that were missed on $\mathrm{ZN}$ staining [20].

The PPV and NPV of ZN gave a value of $70.8 \%$ and $94.2 \%$ respectively while the Auramine O gave $56.82 \%$ and $96.57 \%$. This is in agreement with the report from Turkey where the NPV of ZN was 
lower than the fluorescent staining [15]. The test agreement of $\mathrm{ZN}$ with Culture gave a value of 0.585 Kappa which was lower than the Auoramine $\mathrm{O}$ and PREVI Fluo result. It had a moderate agreement with culture, however there was a substantial agreement of $\mathrm{ZN}$ with PREVI Fluo (kappa $=0.636)$. Although there was substantial agreement between these two methods, still ZN had lower sensitivity.

The rate of false negativity in $\mathrm{ZN}$ was higher than PREVI Fluo and Auramine $\mathrm{O}$ which means the method had high probability to loss true positive cases. However, the false positivity of $\mathrm{ZN}$ was lower than the two methods this suggested that the probability of $\mathrm{ZN}$ to detect non bacilli artifacts was lower than the two methods. The likelihood ratio of $\mathrm{ZN}$ was slightly higher than PREVI Fluo and Auramine O methods which were $0.45,0.25$ and 0.24 respectively. Compare to these two methods $\mathrm{ZN}$ was not a good diagnostic method.

A total of 744 slides were run with Auramine O, spot-morning-spot samples were smeared and stained and from which $17.7 \%$ were positive from the total 248 patients. In a similar study in Kenya [21] overall $23 \%$ of FM smears were positive which was higher than the current study with Auramine O. This could be as result that they used higher sample size in their study (21,104 smears were included in the study). In another study, sensitivity and specificity documented for the different modalities were $84.7 \%$ and $98.9 \%$ [18], respectively, for the LED assessment with Auramine O. The increased sensitivity of Auramine O is greatest in low grade positives. The proportion of low grade positives in the population served may thus determine the relative sensitivity of the method over LM in any setting. This becomes more clear with the finding that the PPV for the Auramine O is $56.82 \%$ while for the $\mathrm{ZN}$ it is $70.83 \%$ (as the PPV gives lower result, it could be an indication that the test could be giving more false positives). One disadvantage of the fluorescent staining technique is that it may sometimes yield false positive results. However, most of these can be prevented by restaining the smear by the ZN method for bright-light microscopy [22].

Apart from these studies, in a study done in kenya, Nairobi, there was no difference in sensitivity and specificity between LED-FM and ZN microscopy [17]. This could be explained by the great experience of study technicians with the $\mathrm{ZN}$ method and the fact that they never used FM before. Also they did not process the culture reference standard for each participant used for microscopy as they were requesting a $4^{\text {th }}$ sample for culture confirmation in which case not all patients were able to produce a $4^{\text {th }}$ specimen for culture and among those who did; the macroscopic appearance of the $4^{\text {th }}$ specimen was of poorer quality than those specimens submitted for microscopy. With regards to its agreement with the kappa scale in relation to the gold standard, culture, Auramine $\mathrm{O}$ in this study has a kappa of 0.621 (substantial agreement) which agrees better than the $\mathrm{ZN}(0.585$, moderate agreement) but still a little less than the PREVI Fluo method (0.664, substantial agreement). In addition the Auramine $\mathrm{O}$ and the PREVI Fluo had a substantial agreement of $\mathrm{K}=0.745$. Auramine $\mathrm{O}$ had a smear positivity of $14 \%$ in HIV positive which was higher than the ZN (9.3\%) but with no significant difference whereas in PREVI Fluo it had a case detection rate of $11.6 \%$.

Concerning the sensitivity and specificity of Auramine $\mathrm{O}$ stain, it had a sensitivity of $78.13 \%$ and a specificity of $91.2 \%$, which is significantly higher in sensitivity than $\mathrm{ZN}$ and lower than the $\mathrm{ZN}$ finding in specificity ( $56.7 \%$ and $96.79 \%$ respectively for $\mathrm{ZN}$ ). This is in agreement with the study in Ethiopia, Nepal, Nigeria, and Yemen [14] using three LED-FM smears per patient resulted in sensitivity of $77.1 \%(73.3 \%-80.6 \%)$. The lower specificity may thus be the impact of the larger amount of operational data collected in this study, at the cost of a less-than-ideal reference standard.

In this study smears were made within a three-specimen set from the same patient and for processed by different techniques. This will ensure the issue of bias associated with which sputum sample is used for which slide, that is morning sputum sample being used for one method alone kind of bias is eliminated. The negative likelihood ratio of Auramine $\mathrm{O}$ was smaller than $\mathrm{ZN}$ and PREVI Fluo methods, which were $0.24,0.45$, and 0.25 respectively. Compared to these two methods Auramine $\mathrm{O}$ was a very good diagnostic method since its negative likelihood ratio was nearest to zero.

In the current study the smear detection rate of PREVI Fluo was found to be $14.1 \%$ while the culture confirmed cases were $12.1 \%$.This is more close to culture results compared to the other two methods (ZN and Auramine O). There was a substantial agreement with PREVI Fluo $\mathrm{TB}$ result and TB Auramine O methods $(\mathrm{K}=0.745)$. Out of the total 34 PREVI Fluo positive cases, $88.6 \%$ had also a positive result with the Auramine $\mathrm{O}$, while only 4 had a discordant result. Furthermore, while $6.1 \%$ of the Auramine O totals (17.7\%) were negative with PREVI Fluo.

The sensitivity of the PREVI Fluo method was $76.67 \%$ better than $\mathrm{ZN}$ and slightly less than Auramine O which were 59.07\% and 78.13\% respectively. Nevertheless, the specificity $(94.5 \%)$ was lower than ZN (96.79\%) and higher than Auramine O (91.2\%). The likely hood Ratio of a negative test of the PREVI Fluo method (0.24) was lower than ZN (0.44) which indicated PREVI Fluo is a better diagnostic test than ZN since its value is closer to zero.

The PREVI Fluo TB method has a PPV of $65.71 \%$ which is lower than the Auramine O (56.82\%) but with a comparable NPV $(96.71 \%$ and $96.57 \%$ respectively). Hence, in the case of any infectious disease with public health implications, it is important to have a test or rule that has a high sensitivity, negative predictive value and Positive predictive value, so that patients with true disease are treated and those with a low possibility of disease can be discharged from the hospital safely without treatment. The rate of false positivity is lower as well than the Auramine O method. In PREVI Fluo TB method the counter staining dye makes the back ground of the smear bright red color which creates a good contrast between the fluorescing bacilli and back ground. The back ground helps the stain to have fewer artifacts. Consequently, even if epithelial and other WBCs are present, the stain can use that as an advantage to give a better background contrast. Furthermore, it helps the technician better to focus and find the field making it easier to differentiate when the objective is out of focus. Subsequently, this could be ideal for the inexperienced technician.

\section{Conclusion}

The PREVI Fluo TB fluorescent staining method had nearly identical sensitivity compared to the conventional Auramine O fluorescent staining method for the detection of AFB in patient specimens. It had a significant higher sensitivity than $\mathrm{ZN}$ staining method, but the specificity was less than ZN. Auramine O also had significantly higher sensitivity than $\mathrm{ZN}$ whereas the specificity was lower.

\section{Acknowledgement}

The financial support obtained from the graduate program of Addis Ababa University, Biomerieux SA and EPHI is acknowledged.

\section{References}

1. Floyd K, Blanc L, Raviglione M, Lee J (2002) Resources required for global tuberculosis control. Sci 295: 2040-41. 
Citation: Solomon S, Gebreselassie S, Yitayew B, Kebede A (2015) Comparative Analysis of Performance of PREVI Fluo TB Method by Using LEDMicroscopy. J Med Microb Diagn 4: 178. doi:10.4172/21610703.1000184

Page 5 of 5

2. Dye C, Watt C, Bleed D (2005) Evolution of tuberculosis control and prospects for reducing tuberculosis incidence, prevalence, and deaths globally. JAMA 293: $2767-2775$

3. Perkins M, Roscigno G, Zumla A (2006) Progress towards countries. Lancet 367: 942-943.

4. WHO (2007a) The use of liquid medium for culture and DST.

5. WHO (2009) Approaches to improve sputum smear microscopy for tuberculosis diagnosis, expert group meeting.

6. WHO (2010a) Fluorescent light emitting diode (led) microscopy for diagnosis of tuberculosis policy statement.

7. Hanscheid $T$ (2008) The future looks bright: low-cost fluorescent microscopes for detection of Mycobacterium tuberculosis and Coccidiae. Trans R Soc Trop Med Hyg 102: 520-521

8. Minion J, Sohn H, Pai M (2009) Light-emitting diode technologies for TB diagnosis: what is on the market?. Expert Rev Med Devices 6: 341-345.

9. Shenai S, Minion J, Vadwai V, Tipnis T, Shetty S (2011) Evaluation of light emitting diode-based fluorescence microscopy for the detection of mycobacteria in a tuberculosis-endemic region. Int J Tuberc Lung Dis 15: 483-488.

10. WHO (2007b) Definition of Smear Positive TB. Geneva: World Health Organization. New WHO policies.

11. Duc TMN (2011) Tuberculosis in HIV-infected individuals in Ho Chi Minh City, Vietnam. Texas Medical Center Dissertations, AAI3459881.

12. Karen RS, Megan H, Vivienne N, Philip CH, Andrew R, et al. (2006) Fluorescence versus conventional sputum smear microscopy for tuberculosis: a systematic review. Lancet Infect Dis 6: 570-81.

13. Masood Z, Mohammad N, Majid K, Ghodsieh A (2008) Comparison of the value of two different sputum staining for diagnosis of acid-fast bacilli. Iranian Journal of Clinical Infectious Diseases 3: 99-102.

14. Luis EC, Najla AS, Lovett L, Mohammed AY, Isabel A, et al. (2011b) LED Fluorescence Microscopy for the Diagnosis of Pulmonary Tuberculosis: A MultiCountry Cross Sectional Evaluation. Plosmedicine, 8.

15. Mustafa U, Gonul A, Sami T (2000) A Comparative Study on the Different Staining Methods and Number of Specimens for the Detection of Acid Fast Bacilli. Mem Inst Oswaldo Cruz 95: 855-858.

16. Onubogu CC, Nwokoye NN, Kunle-Ope CN, Raheem TY (2012) Sensitivity of direct smear microscopy for the diagnosis of TB in high HIV prevalent population. Scientific Research and Essays 7: 593-597.

17. Maryline B, Laramie G, Willie G, Philippe JGR, Laurence B, et al. (2011) Performance of LED-Based Fluorescence Microscopy to Diagnose Tuberculosis in a Peripheral Health Centre in Nairobi. Plos ONE 6.

18. Marais BJ, Wendy B, Katrien P, Anneke C, Elizabeth W, et al. (2008) Use of Light-Emitting Diode Fluorescence Microscopy to Detect Acid-Fast Bacilli in Sputum. Clinical Infectious Dis 47: 203-7.

19. Matee M, Mtei L, Lounasvaara T, Wieland AW, Waddell R, et al. (2008) Sputum microscopy for the diagnosis of HIV-associated pulmonary tuberculosis in Tanzania. BMC Infect Dis 8: 68 .

20. Saroj H, Nita P, Bharti M, Sumit G, Vipin K, et al. (2011) Comparison of zieh neelsen \& auramine o staining methods on direct and concentrated smears in clinical specimens. Indian J Tuberc 58: 72-76.

21. Torrea G, Chakaya J, Mayabi M, Deun AV (2008) Evaluation of the Fluoreslent and fluorescence microscopy blinded rechecking trial, Nairobi, Kenya. Int J Tuberc Lung Dis 12: 658-663.

22. Heifets L (1997) Mycobacteriology laboratory. Clin Chest Med 18: 35-41. 